Original research article

\title{
Clinical and psychological outcome after surgery for lumbar spinal stenosis: A prospective observational study with analysis of prognostic factors
}

\author{
Mauro Dobran, Davide Nasi ${ }^{*}$, Maurizio Gladi, Mattia Marinelli, \\ Fabrizio Mancini, Maurizio Iacoangeli, Massimo Scerrati
}

Department of Neurosurgery, Università Politecnica delle Marche, Umberto I General Hospital, Ancona, Italy

\section{A R T I C L E I N F O}

\section{Article history:}

Received 14 May 2017

Accepted 4 December 2017

Available online 8 December 2017

Keywords:

Lumbar spinal stenosis

Psychological outcome

Depression and anxiety

\begin{abstract}
A B S T R A C T
Background: The identification of psychological risk factors is important for the selection of patients before spinal surgery. Moreover, the effect of surgical decompression in lumbar spinal stenosis (LSS) on psychological outcome is not previously well analyzed.

Aim of paper: to investigate clinical and psychological outcome after surgery for LSS and the effect of depressive symptoms and anxiety on the clinical outcome.

Materials and methods: A total of 25 patients with symptomatic LSS underwent decompressive surgery with or without spinal stabilization were prospectively enrolled in this observational surgery. The Symptom Checklist-90-Revised (SCL-90-R) was used to assess global psychological distress with a summary score termed Global Severity Index (GSI) and single psychological disorders including depression (DEP) and anxiety (ANX). The clinical outcome of surgery was evaluated with the Oswestry Disability Index (ODI) and visual analogue scale (VAS) pain assessment.

Results: Compared with baseline, there was a statistically significant improvement in VAS, ODI and GSI after surgery $(p<0.05)$ in all patients. Univariate analysis revealed that patients with high GSI and anxiety and depression scores had significantly higher ODI and VAS scores in the follow-up with a bad outcome.

Conclusions: Surgery for spinal stenosis was effective to treat pain and disability. In this prospective study baseline global psychological distress, depression and anxiety were associated with poorer clinical outcome.
\end{abstract}

() 2017 Published by Elsevier Sp. z o.o. on behalf of Polish Neurological Society.

\footnotetext{
* Corresponding author at: Department of Neurosurgery, Università Politecnica delle Marche, Umberto I General Hospital, Via Conca \#71, Ancona 60020, Italy.

E-mail address: davidenasi83@gmail.com (D. Nasi).

https://doi.org/10.1016/j.pjnns.2017.12.002

0028-3843/@ 2017 Published by Elsevier Sp. z o.o. on behalf of Polish Neurological Society.
} 


\section{Introduction}

Lumbar spinal stenosis (LSS) is a common spinal disorder that most typically affects middle-aged and older adults. The surgical treatment is usually safe and effective when conservative therapy fails [1]. Still today factors that influence the clinical outcome are controversial [2,3]. Psychological factors are important and previous research have documented an association between depressive symptoms and poorer surgery outcome [4-5-9] while the role of anxiety and global psychological distress are still under debate [6-9]. Furthermore, many authors have documented that depressive symptoms have biological effects on wound healing and pain through neuroendocrine-immune alterations [9].

The knowledge of the patient's psychological asset before surgery would be very helpful to choose supporting therapies in preoperative and mostly in postoperative time [10-16]. This would mean to be able to prepare the patient to the best for the surgical procedure in order to achieve good results.

\section{Aim of paper}

The aim of this prospective observational study was to investigate clinical and psychological outcome after surgery for LSS and the effect of global psychological distress, depressive symptoms and anxiety on surgical outcome. Others clinical and radiological baseline factors were analyzed to assess the impact of neurological and psychological outcome.

\section{Materials and methods}

The study included 25 patients with radiologically and clinically defined LSS who underwent decompressive laminectomy with or without fixation from March 2015 to March 2016. The inclusion criteria were the presence of severe back pain, leg pain and claudication neurogenic with radiographic evidence of dural sac or nerve root compression by degenerative changes. All patients had an history of ineffective responses to conservative treatment for almost six months.

The indication for a dynamic stabilization was preoperative MRI evidence of pathological disc (Pfirmann 2-3-4) at the same level of the planned laminectomy; on the contrary in patients with associated degenerative scoliosis (Schwab classification VB0) and or first grade spondylolisthesis the rigid fixation was preferred $[10,11]$. None of the patients had undergone previous spinal surgery or suffered from infections or tumours.

All patients received both an oral and a written explanation of the questionnaire and signed an informed consent form confirming their agreement to join the present study.

Patient demographics, clinical presentation, neurologic examination, indications for surgery, radiological studies and pre-operative psychological variables were prospectively collected in each case. Patient-reported outcome measures were prospectively obtained via clinical evaluation by independent investigator with a set of questionnaires in the preoperative period (7 days before surgery) and 1, 3 and 12 months after the surgery. Questionnaires included the Symptom Checklist-90-Revised (SCL-90-R), the Oswestry Disability Index (ODI) and visual analogue scale (VAS).

The Symptoms Checklist-90 Revisited (SCL-90-R) consists of a list of 90 symptoms of psychopathology and psychosomatic disorders for which the subject is asked to indicate the extent of suffering over the last seven days, attributing every symptom scored on Likert five-point scale from "not at all" (0 points) to "very much" (4 points).

The tool assesses the presence and severity of psychological distress symptoms in the last week before surgery concerning obsessive-compulsive symptoms, interpersonal sensitivity, hostility, phobic anxiety, paranoid ideation, somatization, anxiety and depression [12].

The arithmetic average of every SCL-90-R score determined the Global Severity Index (GSI) which quantifies the overall psychopathological suffering of the subject. The score of each individual symptom domain has been calculated on the answers to the questions related to the specific domain. The final score is expressed as a deviation from the median of the general population (denoted by 0 ) with respect to a standard deviation $(+1 /-1)$. In the present study, the SCL-90-R was used to assess the domains of depression (DEP) and anxiety (ANX).

Statistical methods included the Student $t$ test or the Mann-Whitney $U$ test with continuous variables, depending on the distribution. Categorical values were compared using a chi-square test. Univariate logistic regression analyses were used to investigate the predictors for the surgical outcome on 1-year follow-up.

Statistical significance was accepted at a $p$ value $<0.05$. Statistical analyses were performed using SPSS version 11.0 for Mac.

\section{4. $\quad$ Results}

Results were obtained in 25 patients, including 14 males (56\%) and 11 females (44\%). The mean age at baseline was 70.4 years (range 50-84 years).

The presence of co-morbidities was calculated using Charlson Co-Morbidity Index with an average value of 4.88 [13]. The surgical strategy (decompressive laminectomy with or without rigid or flexible stabilization) was chosenn line with the data of the literature [14,15]: 14 patients out of 25 were decompressed without stabilization and 11 pts have been stabilized with transpedicular screws and rods (7 with a rigid device and 4 with hybrid device). The number of levels decompressed ranged between a minimum of 1 and a maximum of 4 , with an average value of 2.6 level for each patient. The number of instrumented levels ranged between a minimum of 1 and a maximum of 4 , with an average value of the instrumented levels of 2.27 for patient. The average length of postoperative hospital stay was 6 days.

At baseline, the patients' mean VAS score was 7.4, mean ODI score $49 \%$ and mean GSI score 0.60 .

At admission neurogenic claudication was present in 14 patients out of 25 with a limitation of walking capacity between 5 and $500 \mathrm{~m}$ (mean $134 \mathrm{~m}$ ). All patients presented severe leg pain and among these 11 pts severe back pain. 
Paraesthesia of the lower limbs were present in 16 patients out of 25 and weakness in 10 pts.

MRI baseline parameters evaluated including cross-sectional area of the dural sac and nerve root sedimentation sign $[16,17]$. The average value of the cross-sectional area of the dural sac was $39 \mathrm{~mm}^{2}$ in the most stenotic level. The nerve root sedimentation sign was positive in 16 patients out of 25 and 10 patients had radiological evidence of spondylolisthesis (Table 1).

At one year follow-up, in the group of operated patients the average VAS score improved from 7.4 to 2.4 points $(p<0.05)$, the mean ODI from $49 \%$ to $23 \%(p<0.05)$ and the average GSI improved from 0.60 pre-operatively to 0.22 at 12 months $(p<0.05)$.

All patients were treated with analgesics and anti-inflammatory in the perioperative period. In this series there was not a statistically significant difference in the group treated with decompressive laminectomy alone compared to the group of patients treated with spinal stabilization and decompressive laminectomy. Inside the group of patients treated with laminectomy associated with stabilization, there was not significant differences in term of outcome between the

Table 1 - Baseline characteristics and surgical treatment of patients.

Baseline presentation

Age (mean; range)

Male/Female

Charlson Co-morbidity Index (mean)

Low back pain (number of patients)

Leg pain (number of patients)

Neurogenic claudication (number of patients)

Paraesthesia lower limbs (number of patients)

Weakness lower limbs (number of patients)

Pre-operative VAS (mean)

Pre-operative ODI (mean)

Pre-operative (SCL-90-R) - CSI (mean)

Pre-operative (SCL-90-R) - Depression (mean) 0.55

Pre-operative (SCL-90-R) - Anxiety (mean) 0.45

Nerve root sedimentation sign (number of 16 patients)

The cross-sectional area of the dural sac at the most stenotic level (mean $\mathrm{mm}^{2}$ )

Spondylolisthesis (number of patients)

Decompression/Decompression + rigid

fixation/Decompression + hybrid fixation

(number of patients) patients with rigid system and the patients with hybrid system.

In further analyses, univariate logistic regression was used to examine the preoperative factors independently associated with a poor surgery outcome (defined as ODI $>40 \%$ ) on 1-year follow-up.

Patients with psychological profile characterized by a global severity index (GSI) greater than the standard deviation (+1 ds) of the reference population have a higher correlation with a severe disability ODI (greater than $40 \%$ ) one year after surgery $(p=0.035)$, as well as a lower degree of improvement in symptoms $(p=0.029)$.

One year after surgery, patients with a level of preoperative anxiety and depression greater than $(+1 \mathrm{ds})$ the standard deviation had a poorer clinical outcome with a high ODI score $(p=0.029$ and 0.031$)$ but patients with a level of anxiety lower to $(-1 \mathrm{ds})$ the standard deviation had a moderate disability ( $p=0.012$ ) (Table 2).

As described in the literature, at 12 months follow-up, the presence of a severe lumbar canal stenosis (crosssectional area of dural sac less than $40 \mathrm{~mm}^{2}$ ) correlates significantly $(p=0.027)$ with a poor outcome and ODI greater than $40 \%$.

Finally, the postoperative weakness in lower limbs was significantly related to post-operative depression $(p=0.004)$, anxiety $(p=0.008)$ and global psychological distress $(p=0.041)$ 12 months after surgery compared to patients with no neurological deficit.

\section{Discussion}

The main goals in the surgical treatment of LSS are the treatment of disability, the reduction of pain and finally quality of life improvement [1-17]. Literature shows heterogeneous results after the surgical treatment of spinal stenosis. In the short term follow-up, several prospective studies reported a functional benefit ranging from 60 to $79 \%$ of operated patients [18], in other studies there is a high variability of results in long term outcome [19]. Recently, some studies documented better improvement for pain and walking ability with surgery than conservative treatment only [16-19].

Nowadays, in addition to pain and disability, psychosocial aspects are fundamental for the recovery of patients and they have to be considered to choose the proper surgical treatment. Still today many scales exist to evaluate the clinical outcomes of patients operated for lumbar spinal stenosis (LSS) but

Table 2 - Univariate logistic regression analyses of pre-operative psychological factors on surgical outcome (ODI at 1 years).

\begin{tabular}{|c|c|c|c|}
\hline & Pre-operative (SCL-90-R) - GSI > +1 ds & Pre-operative (SCL-90-R) - GSI $<+1$ ds & $p$ value \\
\hline ODI $>40 \%$ & 3 & 3 & \\
\hline \multirow{2}{*}{ ODI $<40 \%$} & 2 & 17 & 0.035 \\
\hline & Pre-operative (SCL-90-R) - Depression > +1 ds & Pre-operative (SCL-90-R) - Depression $<+1$ ds & \\
\hline ODI $>40 \%$ & 4 & 19 & \\
\hline \multirow[t]{2}{*}{ ODI $<40 \%$} & 1 & 1 & 0.029 \\
\hline & Pre-operative (SCL-90-R) - Anxiety > +1 ds & Pre-operative (SCL-90-R) - Anxiety $<+1$ ds & \\
\hline ODI $>40 \%$ & 3 & 18 & \\
\hline ODI $<40 \%$ & 2 & 2 & 0.031 \\
\hline
\end{tabular}


psychological outcomes and improvement in depressive symptoms and anxiety are not extensively investigated [2-13].

In this study, patient's global psychological distress improved after spinal surgery with statistically significance (average GSI improved from 0.60 pre-operatively to 0.22 at 12 months $(p<0.05)$. Depressive symptoms and anxiety improved after surgery too (respectively mean pre-operative DEP of 0.55 became after 1 years 0.39 , while mean pre-operative ANX of 0.45 became after 1 years 0.11 ).

Few previous studies showed that surgery had a relieving effect on depressive symptoms [5-8]. Urban-Baeza et al. [7] reported that among 29 patients with depressive symptoms in the preoperative period, only 15 retained these symptoms after surgery. Patients with depression after surgery showed poorer improvement in symptoms, disability scores, and walking capacity, whereas patients who recovered from depression showed clinical improvement similar to that in the normal mood group. Similarly, Sinikallio et al. [5] described 45 patients with preoperative depression among a cohort of 96; 22 patients had recovered from depression symptoms at the 2 year follow-up. Generally, satisfaction was lower in patients with persistent depression symptoms.

Adogwa et al. [6] described a cohort of 53 patients who had undergone revision surgery for symptomatic recurrent stenosis. Depression was measured using the Zung Self-Rating Depression Scale. Significant improvement from 37.52 to 49.9 $(p<0.001)$ was noted in the scale, and the authors concluded that an increased preoperative Zung depression score was associated with patient's dissatisfaction 2 years after revision lumbar surgery.

These results combined with those of our study indicating that treatment of depression is very important after surgery to get better functional recovery. Moreover, to the best of our knowledge, we firstly described that surgery may improve anxiety status of patients with LSS.

Preoperative selection of patients with the addition of a psychological examination becomes an important part of modern spinal practice. Literature has focused primarily on measuring psychosocial risk factors in patients with low back pain and in patients undergoing various types of spine surgery. Only a very few studies have focused on identifying psychosocial risk factors in a homogeneous selection of patients such as patients with LSS $[8,9]$.

Patients with psychological profile characterized by a global severity index (GSI) higher than the standard deviation (+1 ds) in reference population have a higher correlation with a severe disability ODI (greater than $40 \%$ ) to one year after surgery $(p=0.035)$, as well as a lower degree of improvement in symptoms $(p=0.029)$.

Even patients with a preoperative anxiety and depression level greater than standard deviation presented a poorer clinical outcome with a high ODI score $(p=0.009)$ one year after surgery.

Several studies have reported that depressive symptoms are associated with less favourable outcomes following the surgical treatment of patients with LSS [4-9]. Sinikallio et al. [5] showed that depressive symptoms in the preoperative and early recovery phase are strong and independent predictors of the self reported surgery outcome at 2-year follow-up. Also Adogwa et al. highlighted that the extent of preoperative depression independently predicts postoperative patient's dissatisfaction. Finally, Urban-Baeza et al. [7] showed that depression symptoms before and after surgery led to worse clinical outcomes and different patient's fulfilled.

The role of anxiety as predictor of poor outcome in LSS was not so clear. One study, in a sample of patients undergoing lumbar surgery for spinal canal stenosis, revealed that increased preoperative anxiety correlates with increased postoperative pain, increased postoperative analgesic requirements, prolonged recovery and hospital stay.

In this scenario, our study highlighted the strong role of anxiety for bad surgical outcome: in fact, $90 \%$ of patients with a level of anxiety lower to the standard deviation have a disability of moderate degree or less 1 years after surgery $(p=0.012)$.

Finally, the presence of motor deficit in the lower limbs was significantly related to post-operative depression $(p=0.004)$, anxiety $(p=0.008)$ and global psychological distress $(p=0.041)$ 12 months after surgery compared to patients with no neurological deficit. This issue strengthens the necessity to perform the surgical treatment as soon as possible in front of neurological deficits.

\section{Conclusions}

1. Surgery for spinal stenosis is effective not only for pain control and disability improvement but also for preoperative psychological symptoms at 1-year follow-up.

2. In our prospective study baseline in patients operated for lumbar spinal stenosis, global psychological distress, depression and anxiety were associated with poorer clinical outcome.

3. The appropriate recognition and treatment of depression and anxiety symptoms before and after spinal surgery could improve clinical results. We are aware that this is a small series but our prospective observational study may be useful to contribute to the preoperative selection of patients with the addition of a psychological examination as an important part of modern spinal practice.

\section{Ethical approval}

All procedures performed in studies involving human participants were in accordance with the ethical standards of the institutional and/or national research committee and with the 1964 Helsinki Declaration and its later amendments or comparable ethical standards.

\section{Informed consent}

Informed consent was obtained from all individual participants included in the study.

\section{Conflict of interest}

None declared. 


\section{Acknowledgement and financial support}

None declared.

\section{REFERENCES}

[1] Machado GC, Ferreira PH, Yoo RI, Harris IA, Pinheiro MB, Koes BW, et al. Surgical options for lumbar spinal stenosis. Cochrane Database Syst Rev 2016;11(November):CD012421.

[2] Budithi S, Dhawan R, Cattell A, Balain B, Jaffray D. Only walking matters-assessment following lumbar stenosis decompression. Eur Spine J 2017;26(February (2)):481-7.

[3] Dobran M, Iacoangeli M, Di Somma LGM, Di Rienzo A, Colasanti R, Nocchi N, et al. Neurological outcome in a series of 58 patients operated for traumatic thoracolumbar spinal cord injuries. Surg Neurol Int 2014;5 (Suppl. 7):S329-32.

[4] Sinikallio S, Aalto T, Airaksinen O, Herno A, Kröger H, Viinamäki H. Depressive burden in the preoperative and early recovery phase predicts poorer surgery outcome among lumbar spinal stenosis patients: a one-year prospective follow-up study. Spine 2009;34(23):2573-8.

[5] Sinikallio S, Aalto T, Airaksinen O, Lehto SM, Kröger H, Viinamäki H. Depression is associated with a poorer outcome of lumbar spinal stenosis surgery: a two-year prospective follow-up study. Spine 2011;36(8):677-82.

[6] Adogwa O, Parker SL, Shau DN, Mendenhall SK, Bydon A, Cheng JS, et al. Preoperative Zung depression scale predicts patient satisfaction independent of the extent of improvement after revision lumbar surgery. Spine J 2013;13 (5):501-6.

[7] Urban-Baeza A, Zárate-Kalfópulos B, Romero-Vargas S, Obil-Chavarría C, Brenes-Rojas L, Reyes-Sánchez A. Influence of depression symptoms on patient expectations and clinical outcomes in the surgical management of spinal stenosis. J Neurosurg Spine 2015;22(1):75-9.

[8] Nechanicka N, Barsa P, Harsa P. Psychosocial factors in patients indicated for lumbar spinal stenosis surgery. J Neurol Surg A Cent Eur Neurosurg 2016;77(September (5)):432-40.

[9] Pakarinen M, Vanhanen S, Sinikallio S, Aalto T, Lehto SM, Airaksinen O, et al. Depressive burden is associated with a poorer surgical outcome among lumbar spinal stenosis patients: a 5-year follow-up study. Spine J 2014;14(October (10)):2392-6.
[10] Dobran M, Nasi D, Brunozzi D, Di Somma L, Gladi M, Iacoangeli $\mathrm{M}$, et al. Treatment of unstable thoracolumbar junction burst fractures: short-segment pedicle fixation with inclusion of fracture level versus long-term instrumentation. Acta Neurochir (Wien) 2016;158(October (10)):1883-9.

[11] Dobran M, Iacoangeli M, Nasi D, Nocchi N, Di Rienzo A, Di Somma L, et al. Posterior titanium screw fixation without debridemenet of infected tissue for the treatment of thoracolumbar spontaneous pyogenic spondylodiscitis. Asian Spine J 2016;10(June (3)):465-71.

[12] Schmitz N, Kruse J, Heckrath C, Alberti L, Tress W. Diagnosing mental disorders in primary care: the General Health Questionnaire (GHQ) and the Symptom Check List (SCL-90-R) as screening instruments. Soc Psychiatr Psychiatr Epidemiol 1999;34(7):360-6.

[13] Dobran M, Iacoangeli M, Nocchi N, Di Rienzo A, di Somma LG, Nasi D, et al. Surgical treatment of cervical spine trauma: our experience and results. Asian J Neurosurg 2015;10(July-September (3)):207-11.

[14] Munting E, Röder C, Sobottke R, Dietrich D, Aghayev E. Patient outcomes after laminotomy, hemilaminectomy, laminectomy and laminectomy with instrumented fusion for spinal canal stenosis: a propensity score-based study from the Spine Tango registry. Eur Spine J 2015;24 (2):358-68.

[15] Schöller K, Alimi M, Cong GT, Christos P, Härtl R. Lumbar spinal stenosis associated with degenerative lumbar spondylolisthesis: a systematic review and meta-analysis of secondary fusion rates following open vs minimally invasive decompression. Neurosurgery 2017;80(March (3)):355-67.

[16] Ogikubo O, Forsberg L, Hansson T. The relationship between the cross-sectional area of the cauda equina and the preoperative symptoms in central lumbar spinal stenosis. Spine 2007;32(13):1423-8.

[17] Barz T, Melloh M, Staub LP, Lord SJ, Lange J, Röder CP, et al. Nerve root sedimentation sign: evaluation of a new radiological sign in lumbar spinal stenosis. Spine 2010;35 (8):892-7.

[18] Amundsen T, Weber H, Nordal HJ, Magnaes B, Abdelnoor M, Lilleâs F. Lumbar spinal stenosis: conservative or surgical management?: a prospective 10-year study. Spine 2000;25 (11):1424-35. discussion 1435-36.

[19] Atlas SJ, Keller RB, Robson D, Deyo RA, Singer DE. Surgical and nonsurgical management of lumbar spinal stenosis: four-year outcomes from the Maine lumbar spine study. Spine 2000;25(5):556-62. 